\title{
Concordance of HER2 Immunohistochemistry and Fluorescence In Situ Hybridization Using Tissue Microarray in Breast Cancer
}

\author{
DANIELA FURRER ${ }^{1,2,3}$, SIMON JACOB $^{4,5,6}$, CHANTAL CARON $^{4,5}$, \\ FRANÇOIS SANSCHAGRIN ${ }^{2,4}$, LOUISE PROVENCHER ${ }^{1,2,4,7}$ and CAROLINE DIORIO ${ }^{1,2,3,4}$ \\ ${ }^{1}$ Cancer Research Center at Laval University, and Departments of ${ }^{3}$ Social and Preventive Medicine, \\ ${ }^{6}$ Molecular Biology, Medical Biochemistry and Pathology, and \\ ${ }^{7}$ Surgery, Faculty of Medicine, Laval University, Quebec City, Canada; \\ ${ }^{2}$ Oncology Axis, CHU of Quebec Research Center, Laval University, Quebec City, Canada; \\ ${ }^{4}$ Deschênes-Fabia Center for Breast Diseases, and ${ }^{5}$ Pathology Service, \\ Saint-Sacrement Hospital, Quebec City, Canada
}

\begin{abstract}
Aim: Immunohistochemistry (IHC) and fluorescence in situ hybridization (FISH) are common methods for assessment of human epidermal growth factor receptor 2 (HER2) in breast cancer. Materials and Methods: In a cohort of 498 consecutive patients with breast cancer, we examined concordance between IHC and FISH for HER2 on tissue microarray (TMA) sections. In a subset of 116 specimens, we examined HER2 concordance from the block used for diagnostics and a randomly-chosen additional block (a proxy of the core biopsy). Results: Overall concordance between both methods on TMA sections was $93.8 \%$ and between HER2, determined on diagnostic and additional blocks, was $93.6 \%$ for IHC and 98.0\% for FISH. Conclusion: Since some cases were discordant, we suggest that both methods be used for HER2 assessment. The lower concordance rate between diagnostic and additional blocks using IHC compared to FISH suggests a greater variability of IHC staining across tumor regions than for FISH results.
\end{abstract}

Human epidermal growth factor receptor 2 (HER2) is a transmembrane tyrosine kinase receptor belonging to the family of epidermal growth factor receptors (EGFR) (1). The protein is encoded by the HER2 (ERBB2) gene, which is located on the long arm of chromosome 17 (17q12-21.32) (2). HER2 gene amplification and protein overexpression,

Correspondence to: Caroline Diorio, Ph.D., Axe Oncologie, Centre de Recherche du Centre Hospitalier Universitaire de Québec, Hôpital du Saint-Sacrement, 1050 chemin Ste-Foy, Quebec City, QC G1S 4L8, Canada. Tel: +1 4186827511 ext. 84726, Fax: +1 4186827949, e-mail: caroline.diorio@crchudequebec.ulaval.ca

Key Words: Breast neoplasms, HER2 status, multiple blocks, ASCO/CAP guidelines, biopsy, trastuzumab. which occur in 15 to $20 \%$ of patients with breast cancer, are important markers for poor prognosis, including a more aggressive disease and a shorter survival (3). Moreover, HER2-positive status is considered a predictive marker of response to HER2-targeted drugs, including trastuzumab and lapatinib (4). Given its prognostic, predictive and therefore therapeutic implications, an accurate evaluation of HER2 status is crucial for identification of patients who would most likely benefit from targeted anti-HER2 therapies.

Currently, there are several Food and Drug (FDA)approved methods for the evaluation of HER2 status in breast cancer specimens, including immunohistochemical (IHC) determination of HER2 protein expression or assessment of HER2 gene amplification using in situ hybridization (ISH), most commonly fluorescent ISH (FISH) $(5,6)$. Patients are eligible for targeted anti-HER2 therapies when their breast cancer specimens overexpress the protein at IHC and are HER2-gene amplified at ISH. Since both IHC and FISH present advantages and disadvantages, there is still no consensus on which method is superior for assessing the HER2 status in breast cancer specimens (7). In 2013, the American Society of Clinical Oncology/College of American Pathologists (ASCO/CAP) updated the guidelines published in 2007 to clarify the recommendations for HER2 testing in breast cancer specimens (5). In particular, the 2013 ASCO/CAP guidelines include new scoring criteria for IHC and FISH. Moreover, the updated guidelines recommend performing an initial test (IHC or ISH) on core biopsy. If test results are equivocal, reflex testing on tumor specimen section with an alternative assay (IHC or ISH) should be carried out. In addition, repeat-testing should be performed if there is an apparent histopathological discordance with the test result (5). The $2007 \mathrm{ASCO} / \mathrm{CAP}$ guidelines recommended to perform HER2 testing on resection specimens and to retest when results were equivocal (6). 
Several publications have demonstrated a very good concordance between the results obtained by IHC and FISH for the determination of HER2 status in breast cancer specimens $(8,9)$. Other studies, however, have reported up to $13 \%$ of FISH-positive cases among those scoring negative by IHC (10-15). Considering this discordance, it would therefore be interesting to perform both IHC and FISH on every specimen. Since the vast majority of studies published to date analyzed the concordance between IHC and FISH according to the 2007 ASCO/CAP criteria (6), it is pertinent to analyze the concordance between these two FDA-approved techniques according to the recent ASCO/CAP scoring criteria (5). Moreover, we wished to evaluate the new approach by which HER2 status is determined firstly on core biopsy as recommended by the updated ASCO/CAP guidelines (5).

Tissue microarray (TMA) allows for molecular characterization of large numbers of specimens by means of arranging tissue cores from multiple samples into an empty paraffin block. Large numbers of specimens can therefore be processed under identical conditions and analyzed simultaneously $(16,17)$. The goal of this study was to analyze the concordance of HER2 status determined by IHC and FISH in 498 consecutive breast cancer specimens on TMA sections and evaluate the impact of the new ASCO/CAP scoring guidelines on the concordance between results. Moreover, in a subset of 116 breast cancer specimens, we aimed to evaluate the concordance between HER2 status from the routine diagnostic paraffin block and a randomly chosen additional block (a proxy of the core biopsy) using TMA.

\section{Materials and Methods}

Specimen collection and patient population. The study population has already been described elsewhere (18). Briefly, formalin-fixed, paraffin-embedded breast cancer tissues from 554 consecutive patients with invasive breast carcinoma were used. Patients who received neoadjuvant chemotherapy or with a tumor size smaller than $1 \mathrm{~cm}$ on histological slides were excluded from the study. All samples were collected at the Centre des Maladies du Sein Deschênes-Fabia at the Saint-Sacrement Hospital in Québec, Québec, Canada, between February 2011 and April 2012. ASCO/CAP recommendations regarding formalin fixation time and time to fixative were followed (5). Written informed consent was obtained from all participants. Ethical approval of the study was obtained from the Research Ethics Committee of the Centre de Recherche du CHU de Québec (Permit Number: DR-002-1286).

TMA construction. TMAs were constructed as previously described (18). Briefly, for the 554 consecutive breast cancer specimens, the most representative tissue block from each case was selected by the pathologist for HER2 assessment by IHC and FISH. Two tumoral regions showing the strongest IHC staining were delineated on the IHC slide by the pathologist. Four $0.6 \mathrm{~mm}$ tissue cylinders were punched using a manual arraying instrument within these delineated regions (Beecher Instruments, Silver Spring, MD, USA) and were inserted into empty recipient paraffin blocks. We call these TMA blocks "diagnostic TMA". Among the 554 cases present on the diagnostic TMA, cases that had at least two additional paraffin blocks showing the same histological type as the paraffin block that was used for the construction of the diagnostic TMA were selected. Among these cases, 100 cases were randomly chosen. Suitable blocks were identified using $\mathrm{H} \& \mathrm{E}$ section and two tumoral regions were delineated without previous IHC staining on the H\&E section by two trained technologists and verified by a pathologist. Four 0.6 $\mathrm{mm}$ tissue cylinders were then punched from these regions. Since we also performed an oversampling of HER2-positive cases (16 cases that were scored as HER2-positive on diagnostic TMA section but that had not been randomly selected were added to the 100 cases), a total of 116 cases were inserted into an empty paraffin block. We call these TMA blocks "random TMA". Consecutive 4$\mu \mathrm{m}$ sections were processed by IHC and FISH. From each TMA block one section was stained with H\&E for reference histology.

Immunohistochemistry. HER2 protein expression was performed using the FDA-approved HercepTest ${ }^{\mathrm{TM}}$ kit (DAKO Diagnostics, Glostrup, Denmark) on an automated immunostaining system (Autostainer; DAKO), according to the manufacturer's instructions.

FISH. HER2 gene copy number was evaluated using the FDAapproved Path Vysion ${ }^{\mathrm{TM}}$ HER2 DNA Probe kit (Abbott Molecular, Des Plaines, IL, USA/Inter Medico, Markham, Canada), according to the manufacturer's instructions.

HER2 evaluation on TMA. A core was considered satisfactory for analysis if tumor tissue occupied $>10 \%$ of the core area. Immunohistochemical staining was analyzed visually. Cytoplasmic staining was ignored and only invasive tumor was scored. Fluorescent signals were evaluated as previously described (18). Briefly, fluorescent signals were evaluated with an epifluorescence microscope (Axio Imager M1; Zeiss, Göttingen, Germany). Automated analysis of fluorescence signals was carried out using the FDA-approved MetaSystems ${ }^{\mathrm{TM}}$ image analysis system (19). For cores with equivocal results, automated image analysis was followed by manual counting in at least 60 nonoverlapping tumor cells. Moreover, manual counting was performed in 40 nonoverlapping tumor cells when: the average HER 2 copy number per tile was $\geq 4.0$ and $\leq 6.0$ at the automated image analysis; automated counting of signals from the invasive cancer cells was impeded (low cellular density or high stromal density); polysomy or monosomy of chromosome 17 as defined by Tubbs and collaborators (20) was suspected. In addition, all cores with ratio $>1.5$ and $<3.0$ at the automated analysis were visually verified. Each informative core was evaluated separately in a blind fashion. Cores were analyzed by trained technologists, and all results were validated by breast pathologists. Average results of informative cores were considered. IHC and FISH results were reported according to the 2013 ASCO/CAP guidelines (5).

Statistical analysis. Only cases with at least one informative core for IHC or FISH were included in the analysis. The concordance between IHC and FISH was analyzed by comparing average IHC staining and average gene amplification results. Averages of HER2 status by IHC and FISH on diagnostic TMA sections were available for 498 out of the 554 total cases $(89.9 \%)$. The average HER2 status by IHC on diagnostic TMA and random TMA were available for 106 cases out of the 116 selected cases $(91.4 \%)$, whereas those by 
FISH on diagnostic TMA and random TMA were available for $87.9 \%$ of cases (102/116). Average HER2 status by IHC and FISH on random TMA sections were available for 99 cases out of the 116 selected cases (85.3\%). In order to evaluate the agreement between the two methods, positive, negative and overall concordance were calculated as previously published (18) following the recommendation of the $2013 \mathrm{ASCO} / \mathrm{CAP}$ guidelines (5). The level of agreement was also measured using the Cohen's kappa test (agreement adjusted by chance). All analyses were performed using SAS software (version 9.1.3; SAS Institute, Inc., Cary, NC, USA).

\section{Results}

Concordance of HER2 status determined by IHC and FISH on diagnostic TMA section is summarized in Table I. The overall agreement between the two methods was $93.8 \%$ (kappa value $=0.60$ ). The positive agreement and the negative agreements were $69.2 \%$, and $92.9 \%$, respectively. Among the 423 cases considered as FISH non-amplified, protein overexpression was observed in one $(0.2 \%)$ case. Five $(7.7 \%)$ out of the 65 cases that showed gene amplification were negative at IHC.

We also assessed the concordance rates between HER2 status determined by IHC and FISH on TMA section according to the number of informative cores per case (one or two vs. three or four cores). The overall, positive and negative agreements for cases with one or two informative cores were lower $(85.7 \%, 58.3 \%$ and $90.3 \%$, respectively) than for cases with three or four evaluable cores $(91.9 \%$, $75.6 \%$ and $94.3 \%$, respectively).

Table II shows the concordance rate of HER2 status determined by IHC and FISH on random TMA section. The overall agreement was $83.5 \%$ (kappa value $=0.67$ ). The positive and negative agreements were $71.4 \%$, and $90.3 \%$, respectively. Among the 62 cases considered FISH nonamplified, none was evaluated as $3+$ for protein overexpression. Of the 35 cases that showed gene amplification, two $(5.7 \%)$ were negative at IHC.

Concordance of HER2 status determined by FISH on diagnostic TMA section and random TMA section is summarized in Table III. In this analysis, we observed an overall agreement of $98.0 \%$. The positive and negative agreements were $97.2 \%$, and $98.5 \%$, respectively. Of the 66 cases considered FISH non-amplified on diagnostic TMA section, one $(1.5 \%)$ was evaluated as equivocal on random TMA section. One (2.8\%) out of the 36 cases that showed gene amplification on diagnostic TMA section was equivocal for HER2 gene amplification on random TMA section.

Table IV presents the concordance of HER2 status determined by IHC on diagnostic TMA section and random TMA section. The overall agreement between the two methods was $93.6 \%$ (kappa value $=0.86$ ). The positive agreement and the negative agreements were $92.9 \%$, and $93.9 \%$, respectively. Among the 66 cases considered negative
Table I. Concordance of human epidermal growth factor receptor 2 (HER2) status determined by immunohistochemistry (IHC) and fluorescence in situ hybridization (FISH) on diagnostic tissue microarray (TMA) section according to the 2013 American Society of Clinical Oncology/College of American Pathologists scoring system.

\begin{tabular}{lcccr}
\hline & \multicolumn{4}{c}{ FISH TMA, n } \\
\cline { 2 - 5 } IHC TMA & Non-amplified & Equivocal & Amplified & Total \\
\hline $0-1+$ & 393 & 8 & 5 & 406 \\
$2+$ & 29 & 2 & 15 & 46 \\
$3+$ & 1 & 0 & 45 & 46 \\
Total & 423 & 10 & 65 & 498 \\
\hline
\end{tabular}

Overall agreement: $93.8 \%$; sensitivity: $69.2 \%$; specificity: $92.9 \%$; kappa value $=0.60$; kappa value when equivocal cases are excluded $=0.93$.

Table II. Concordance of human epidermal growth factor receptor 2 (HER2) status determined by immunohistochemistry (IHC) and fluorescence in situ hybridization (FISH) on random tissue microarray (TMA) section according to the 2013 American Society of Clinical Oncology/College of American Pathologists scoring system.

\begin{tabular}{|c|c|c|c|c|}
\hline \multirow{2}{*}{$\begin{array}{l}\text { IHC random } \\
\text { TMA section }\end{array}$} & \multicolumn{4}{|c|}{ FISH random TMA section, $n$} \\
\hline & Non-amplified & Equivocal & Amplified & Total \\
\hline $0 / 1+$ & 56 & 1 & 2 & 59 \\
\hline $2+$ & 6 & 1 & 8 & 15 \\
\hline $3+$ & 0 & 0 & 25 & 25 \\
\hline Total & 62 & 2 & 35 & 99 \\
\hline
\end{tabular}

Overall agreement: $83.5 \%$; sensitivity: $71.4 \%$; specificity: $90.3 \%$; kappa value $=0.67$; kappa value when equivocal cases are excluded $=0.94$.

for protein overexpression on diagnostic TMA section, four $(6.1 \%)$ were evaluated as equivocal on random TMA section. Two out of the 28 cases evaluated as positive for protein overexpression on diagnostic TMA section were considered equivocal on random TMA section.

\section{Discussion}

We observed a moderate concordance rate between the HER2 status determined by IHC and FISH on TMA section in 498 consecutive breast cancer specimens. In our hands, we recorded 93.8\% concordance (kappa value of 0.60 ) between the two techniques when $2013 \mathrm{ASCO} / \mathrm{CAP}$ scoring criteria were used. Similar studies performed on breast cancer specimens using TMA section reported concordance rates ranging from $78.1 \%$ to $98.0 \%$ between the HER2 status obtained by IHC and FISH (21-27). The concordance results observed in those studies were evaluated using the 2007 ASCO/CAP scoring criteria (6). When we analyzed our data 
Table III. Concordance of human epidermal growth factor receptor 2 (HER2) status determined by fluorescence in situ hybridization (FISH) on diagnostic tissue microarray (TMA) section and random TMA section according to the 2013 American Society of Clinical Oncology/College of American Pathologists scoring system.

\begin{tabular}{lcccr}
\hline & \multicolumn{3}{c}{ FISH diagnostic TMA section, $\mathrm{n}$} \\
\cline { 2 - 5 } FISH random section & Non-amplified & Equivocal & Amplified & Total \\
\hline Non-amplified & 65 & 0 & 0 & 65 \\
Equivocal & 1 & 0 & 1 & 2 \\
Amplified & 0 & 0 & 35 & 35 \\
Total & 66 & 0 & 36 & 102 \\
\hline
\end{tabular}

Overall agreement: $98.0 \%$; sensitivity: $97.2 \%$; specificity: $98.5 \%$; kappa value when equivocal cases are excluded $=1.00$.

Table IV. Concordance of human epidermal growth factor receptor 2 (HER2) status determined by immunohistochemistry (IHC) on diagnostic tissue microarray (TMA) section and random TMA section according to the 2013 American Society of Clinical Oncology/College of American Pathologists scoring system.

\begin{tabular}{lrrrr}
\hline & \multicolumn{4}{c}{ IHC diagnostic TMA section, $\mathrm{n}$} \\
\cline { 2 - 5 } IHC random & \multicolumn{1}{c}{$2+$} & $3+$ & Total \\
\hline TMA section & $0 / 1+$ & 2 & 0 & 64 \\
$2+1+$ & 62 & 10 & 2 & 16 \\
$3+$ & 4 & 0 & 26 & 26 \\
Total & 0 & 12 & 28 & 106 \\
\hline
\end{tabular}

Overall agreement: 93.6\%; sensitivity: 92.9\%; specificity: $93.9 \%$; kappa value $=0.86$; kappa value when equivocal cases are excluded $=1.00$.

using the 2007 ASCO/CAP scoring criteria, overall concordance was $91.5 \%$ (453/495) (sensitivity was $64.1 \%$ (of the 64 amplified cases, six were IHC $0 / 1+, 17$ were IHC $2+$, and 41 were IHC $3+$ ), specificity was $95.6 \%$ (of the 431 non amplified cases, 412 were IHC $0 / 1+, 18$ were IHC $2+$ and one was IHC 3+) and kappa value was 0.66), within the concordance range reported in the above mentioned studies. Among the 431 cases considered FISH non-amplified, one $(0.2 \%)$ was evaluated as positive for protein expression. Six $(9.4 \%)$ of the 64 cases that showed gene amplification were negative at IHC.

To our knowledge, this is the first study that analyzed the concordance between IHC and FISH on TMA section in breast cancer specimens according to the 2007 and 2013 ASCO/CAP criteria. To date, only one study analyzed the concordance between IHC and FISH on whole-tissue sections in 189 non-consecutive breast cancer specimens using both ASCO/CAP scoring criteria (28). Similar to
Garbar and collaborators, we recorded a decrease in the percentage of cases evaluated as FISH-amplified and IHCnegative when the 2013 ASCO/CAP scoring criteria were used. However, Garbar et al. reported one case considered FISH-non-amplified and IHC-positive using the 2013 ASCO/CAP scoring criteria versus no cases using the 2007 ASCO/CAP scoring criteria, whereas in our study, the utilization of the recent ASCO/CAP scoring criteria did not change the percentage of these cases.

We recorded $7.7 \%$ of amplified cases among those considered negative at IHC $(9.4 \%$ when 2007 ASCO/CAP scoring criteria were used). Similar studies comparing the concordance between IHC and FISH on TMA section reported between $1.24 \%$ and $11.5 \%$ of IHC negative cases being FISHamplified (21-23, 25, 27). In our study, one non-amplified case was considered HER2-overexpressing with IHC, representing thus $0.23 \%$ of the IHC-positive cases (same proportion when 2007 ASCO/CAP guidelines were used). Similar studies have reported discordance rates for this category ranging from $0 \%$ to $23.5 \%(21-23,25)$. We noted that this discordant case showed polysomy of chromosome 17 . In agreement with previous studies $(21,26,27)$, we postulate that the presence of chromosome 17 aneusomy could at least in part explain the inconsistence between results.

A goal of our project was also to evaluate the impact of the new ASCO/CAP guidelines on the evaluation of HER2 status. The updated guidelines recommend performing an initial test (IHC or ISH) on core biopsy and specimens should be retested with an alternative assay when results are equivocal (5). Since IHC and FISH were not routinely performed on core biopsy for the 554 consecutive breast cancer specimens, for 116 selected breast cancer specimens we performed IHC and FISH on an additional paraffin block, randomly chosen from among all paraffin blocks of the same specimen that presented the same histological type as the routine diagnostic paraffin block, i.e. the paraffin block that was used to make the diagnosis. Since this additional block was randomly chosen, we consider this block a proxy of the core biopsy performed by the radiologist under ultrasound control (that is a random selection of a tumor area). We then compared IHC and FISH results obtained on the random TMA section (which simulate the HER2 status that would be obtained when IHC and FISH were performed on core biopsy) with IHC and FISH results obtained on diagnostic TMA section (which represent results obtained on excisional breast cancer specimens). We recorded an excellent overall concordance $(98.0 \%$, kappa value $=0.94)$ between the HER2 gene amplification status observed on diagnostic TMA section and on the random TMA section. The overall concordance rate between HER2 protein overexpression obtained on diagnostic TMA section and random TMA section, however, was $93.6 \%$, and therefore does not fulfil the ASCO/CAP suggestion of concordance greater than $95 \%$ 
for clearly negative and positive cases (5). Overall concordance rates between IHC and FISH performed on random TMA section and on diagnostic TMA section were also both lower than $95 \%$ ( $83.5 \%$ vs. $93.8 \%)$.

In a similar study conducted on a cohort of 139 breast cancer cases for which HER2 status was determined in more than one block of a single tumor focus, Bethune et al. analyzed the HER2 concordance between the different blocks (both determined on whole tissue section) and reported 96.4\% concordance (29). Results were considered concordant when the final HER2 status of all blocks was the same, regardless of whether HER2 status was determined by IHC or FISH. Selected cases of our cohort are comparable to those of the mentioned study, since blocks were from different tumor foci for only one patient of the subset. When HER2 status was defined according to the combined IHC and FISH results, we found $93.7 \%$ (89/95) concordance between diagnostic block and randomly selected block [sensitivity was $94.6 \%$; of the 37 cases evaluated as positive on the diagnostic TMA section, two were considered equivocal and 35 positive on the random TMA section), specificity was $93.1 \%$ (of the 58 cases considered negative on the diagnostic TMA section, 54 were considered negative, and four equivocal on the random TMA section) and the kappa-value was 0.85]. This difference might be explained by the fact that Bethune et al. used the former ASCO/CAP scoring guidelines. Similar to Bethune et al., we observed that the majority of discordant cases (seven out of nine) displayed an equivocal HER2 immunostaining. Unlike our study, Bethune et al. did not differentiate whether the concordance rate between the blocks varied according to which test had been used to determine HER2 status (IHC or FISH), as in their study HER2 status was determined by IHC, and FISH had mainly been performed on specimens with equivocal IHC staining. In our hands, HER2 concordance between the different blocks was higher when HER2 status was determined by FISH than by IHC. A study performed on a cohort of breast cancer specimens with equivocal immunostaining (evaluated on whole-tissue section according to the scoring guidelines of the HercepTest) reported that $68 \%$ of cases had a different score when the IHC staining was performed on additional blocks from the same breast tumor specimen or from axillary lymph node metastasis (30). This discordance in immunological staining between different blocks was substantially higher compared to that in our study. This difference may be explained by the fact that Lewis et al. analyzed the concordance for IHC scoring exclusively in cases that were originally evaluated as equivocal at IHC. The interpretation of immunostaining being based on semi-quantitative scoring, reported that interobserver variability and therefore discrepancies in HER2 IHC results were particularly elevated for cases scoring $2+(31)$.
Analogous studies performed on multifocal and multicentric breast cancer specimens have analyzed concordance of HER2 status (determined on whole-tissue section) between different blocks from different tumor foci (32-34). Compared to our results, two studies had observed slightly lower concordance rate in HER2 gene amplification determined on different blocks, ranging from $90.3 \%$ to $94 \%$ $(33,34)$. These studies, however, reported their results using the 2007 ASCO/CAP guidelines (34) or other criteria for HER2 gene amplification (33). Another study reported 93.5\% concordance rate in HER2 status determined by IHC and FISH from different blocks, where the largest focus displayed the most positive result in $98.4 \%$ of cases (32). This latter study, however, did not compare IHC and FISH results separately.

In contrast to our study, an analogous study that compared HER2 status determined on the needle core biopsy and subsequent excisional biopsy (whole tissue section) of the same tumor by IHC and FISH observed a higher concordance rate when HER2 status was evaluated by IHC (98\% vs. 92\%) (35).

TMA technology has several advantages over the traditional method, including batch variability reduction and decrease in reagent and technical time required for staining $(16,17,36$, 37). The amount of tissue needed is also reduced (37). In addition, visual assessment of immunohistochemical staining might be easier on TMA slides comparatively to whole tissue sections as the evaluator can compare staining intensities from different specimens on the same TMA slide (38).

Although TMAs represent a useful tool for rapid and efficient examination of large numbers of tumor tissues, this technique presents some limitations, including the training of highly qualified technicians and core losses $(16,38)$. Furthermore, it has been criticized that TMA sections may not accurately represent histopathological characteristics of the whole-tissue section. However, we and others have demonstrated that even one to two $0.6 \mathrm{~mm}$ cores per case can reliably reproduce results achieved on whole-tissue sections $(18,39)$.

\section{Conclusion}

In conclusion, whereas HER2 gene amplification seems to be very constant in different tumor regions, we observed greater variability regarding HER2 IHC staining. Indeed, we recorded a very good concordance (98\%) between HER2 gene amplification status determined on diagnostic TMA section and on random TMA section. Concordance between HER2 protein overexpression observed on diagnostic TMA section and on random TMA section was $93.6 \%$, lower than the $95 \%$ concordance suggested by the ASCO/CAP. The recent guidelines for HER2 testing in breast cancer developed by the ASCO/CAP recommend effectuating an initial test with IHC or ISH in core biopsy specimen. If test 
results are equivocal, reflex testing on tumor specimen section with an alternative assay (IHC or ISH) should be performed. The guidelines do not recommend retesting when the assay result is clearly negative or positive. Nonetheless, based on our observation, we suggest that HER2 status determined on core biopsy by IHC should be interpreted carefully and a confirmatory repeat test using ISH methods on core biopsy or on a tumor specimen section should be considered. In our hands, the FISH method allowed for reliable evaluation of HER2 status on core biopsy.

\section{Conflicts of Interest and Funding}

This work was supported by Hoffmann-La Roche Limited. The funding source had no role in study design, data collection and analysis, decision to publish, or preparation of the manuscript.

\section{Acknowledgements}

The Authors express special thanks to Drs. Anne Choquette, Michel Beauchemin, Mohamed Amin Hashem, Sophie Laberge, Mohib Morcos, Nathalie Mourad, Alexandre Odashiro, and Ion Popa. The Authors are grateful to the personnel of the Service de Pathologie, especially to Céline Plourde, for their precious technical support. DF received doctoral fellowships from the Fonds de recherche du Québec - Santé (FRQS) and the Laval University Cancer Research Center. CD is a recipient of the Canadian Breast Cancer FoundationCanadian Cancer Society Capacity Development award (award \#703003) and the FRQS Research Scholar. This study was supported by the Fondation des Hôpitaux Enfant-Jésus - StSacrement. Clinical specimens were provided by the Fondation du cancer du sein du Québec and the Banque de tissus et de données of the Réseau de recherche sur le cancer of the FRQS, which is affiliated with the Canadian Tumour Repository Network.

\section{References}

1 Yarden Y and Sliwkowski MX: Untangling the ERBB signalling network. Nat Rev Mol Cell Biol 2(2): 127-137, 2001.

2 Popescu NC and Mark GE: Localization of the pks gene, a RAF related sequence on human chromosomes $\mathrm{x}$ and 7 . Oncogene 4(4): 517-519, 1989.

3 Soerjomataram I, Louwman MW, Ribot JG, Roukema JA and Coebergh JW: An overview of prognostic factors for long-term survivors of breast cancer. Breast Cancer Res Treat 107(3): 309330, 2008

4 Esteva FJ, Yu D, Hung MC and Hortobagyi GN: Molecular predictors of response to trastuzumab and lapatinib in breast cancer. Nat Rev Clin Oncol 7(2): 98-107, 2010.

5 Wolff AC, Hammond ME, Hicks DG, Dowsett M, McShane LM, Allison KH, Allred DC, Bartlett JM, Bilous M, Fitzgibbons P, Hanna W, Jenkins RB, Mangu PB, Paik S, Perez EA, Press MF, Spears PA, Vance GH, Viale G, Hayes DF, American Society of Clinical $\mathrm{O}$ and College of American P: Recommendations for human epidermal growth factor receptor 2 testing in breast cancer: American Society of Clinical Oncology/College of American Pathologists Clinical Practice Guideline update. J Clin Oncol 31(31): 3997-4013, 2013.
6 Wolff AC, Hammond ME, Schwartz JN, Hagerty KL, Allred DC, Cote RJ, Dowsett M, Fitzgibbons PL, Hanna WM, Langer A, McShane LM, Paik S, Pegram MD, Perez EA, Press MF, Rhodes A, Sturgeon C, Taube SE, Tubbs R, Vance GH, van de Vijver M, Wheeler TM, Hayes DF and American Society of Clinical Oncology/College of American P: American Society of Clinical Oncology/College of American Pathologists guideline recommendations for human epidermal growth factor receptor 2 testing in breast cancer. Arch Pathol Lab Med 131(1): 18-43, 2007.

7 Furrer D, Sanschagrin F, Jacob S and Diorio C: Advantages and disadvantages of technologies for HER2 testing in breast cancer specimens. Am J Clin Pathol 144(5): 686-703, 2015.

8 Ahmed SS, Iqbal J, Thike AA, Lim AS, Lim TH, Tien SL and Tan PH: HER2/neu revisited: Quality and interpretive issues. J Clin Pathol 64(2): 120-124, 2011.

9 Arnould L, Roger P, Macgrogan G, Chenard MP, Balaton A, Beauclair S and Penault-Llorca F: Accuracy of HER2 status determination on breast core-needle biopsies (immunohistochemistry, FISH, CISH and SISH vs. FISH). Mod Pathol 25(5): 675-682, 2012.

10 Bahreini F, Soltanian AR and Mehdipour P: A meta-analysis on concordance between immunohistochemistry (IHC) and fluorescence in situ hybridization (FISH) to detect HER2 gene overexpression in breast cancer. Breast Cancer 22(6): 615-625, 2014.

11 Kovacs A and Stenman G: HER2-testing in 538 consecutive breast cancer cases using FISH and immunohistochemistry. Pathol Res Pract 206(1): 39-42, 2010.

12 Lal P, Salazar PA, Hudis CA, Ladanyi M and Chen B: Her-2 testing in breast cancer using immunohistochemical analysis and fluorescence in situ hybridization: A single-institution experience of 2,279 cases and comparison of dual-color and single-color scoring. Am J Clin Pathol 121(5): 631-636, 2004.

13 Martin V, Camponovo A, Ghisletta M, Bongiovanni M and Mazzucchelli L: Internal quality assurance program for ERBB2 (HER2) testing improves the selection of breast cancer patients for treatment with trastuzumab. Patholog Res Int 2012: 261857, 2012.

14 Owens MA, Horten BC and Da Silva MM: Her2 amplification ratios by fluorescence in situ hybridization and correlation with immunohistochemistry in a cohort of 6556 breast cancer tissues. Clin Breast Cancer 5(1): 63-69, 2004.

15 Prati R, Apple SK, He J, Gornbein JA and Chang HR: Histopathologic characteristics predicting HER2/neu amplification in breast cancer. Breast J 11(6): 433-439, 2005.

16 Camp RL, Charette LA and Rimm DL: Validation of tissue microarray technology in breast carcinoma. Lab Invest $80(12)$ : 1943-1949, 2000.

17 Gillett CE, Springall RJ, Barnes DM and Hanby AM: Multiple tissue core arrays in histopathology research: A validation study. J Pathol 192(4): 549-553, 2000.

18 Furrer D, Jacob S, Caron C, Sanschagrin F, Provencher L and Diorio C: Tissue microarray is a reliable tool for the evaluation of HER2 amplification in breast cancer. Anticancer Res 36(9): 4661-4666, 2016.

19 Tubbs RR, Pettay JD, Swain E, Roche PC, Powell W, Hicks DG and Grogan T: Automation of manual components and image quantification of direct dual label fluorescence in situ hybridization (FISH) for HER2 gene amplification: A feasibility study. Appl Immunohistochem Mol Morphol 14(4): 436-440, 2006. 
20 Tubbs RR, Hicks DG, Cook J, Downs-Kelly E, Pettay J, Hartke MB, Hood L, Neelon R, Myles J, Budd GT, Moore HC, Andresen S and Crowe JP: Fluorescence in situ hybridization (FISH) as primary methodology for the assessment of HER2 status in adenocarcinoma of the breast: A single-institution experience. Diagn Mol Pathol 16(4): 207-210, 2007.

21 Egervari K, Szollosi Z and Nemes Z: Tissue microarray technology in breast cancer HER2 diagnostics. Pathol Res Pract 203(3): 169-177, 2007.

22 Hwang CC, Pintye M, Chang LC, Chen HY, Yeh KY, Chein HP, Lee $\mathrm{N}$ and Chen JR: Dual-colour chromogenic in situ hybridization is a potential alternative to fluorescence in situ hybridization in HER2 testing. Histopathology 59(5): 984-992, 2011.

23 Lee Y, Ryu Y, Jeong H, Chang H, Kim Y and Kim A: Effectiveness of silver-enhanced in situ hybridization for evaluating HER2 gene status in invasive breast carcinoma: A comparative study. Arch Med Res 43(2): 139-144, 2012.

24 O'Grady A, Flahavan CM, Kay EW, Barrett HL and Leader MB: HER2 analysis in tissue microarrays of archival human breast cancer: Comparison of immunohistochemistry and fluorescence in situ hybridization. Appl Immunohistochem Mol Morphol 11(2): 177-182, 2003.

25 Park K, Han S, Kim JY, Kim HJ, Kwon JE and Gwak G: Silverenhanced in situ hybridization as an alternative to fluorescence in situ hybridization for assaying HER 2 amplification in clinical breast cancer. J Breast Cancer 14(4): 276-282, 2011.

26 Saez A, Andreu FJ, Segui MA, Bare ML, Fernandez S, Dinares $\mathrm{C}$ and Rey M: HER2 gene amplification by chromogenic in situ hybridisation (CISH) compared with fluorescence in situ hybridisation (FISH) in breast cancer - a study of two hundred cases. Breast 15(4): 519-527, 2006.

27 Schiavon BN, Jasani B, de Brot L, Vassallo J, Damascena A, Cirullo-Neto J, Ivanildo Neves J, Augusto Soares F, Gobbi H and Malagoli Rocha R: Evaluation of reliability of FISH versus brightfield dual-probe in situ hybridization (BDISH) for frontline assessment of HER2 status in breast cancer samples in a community setting: Influence of poor tissue preservation. Am J Surg Pathol 36(10): 1489-1496, 2012.

28 Garbar C, Savoye AM, Mascaux C, Brabencova E and Cure H: The human epidermal growth factor receptor 2 screening tests for breast cancer suggested by the new updated recommendation of the American Society of Clinical Oncology/College of American Pathologists will involve a rise of the in situ hybridization tests for the European laboratories of pathology. ISRN Oncol 2014: 793695, 2014.

29 Bethune GC, Mullen JB and Chang MC: Detecting intratumoral heterogeneity in routine breast-HER2 testing: Low yield of testing multiple blocks. Ann Diagn Pathol 19(6): 385-390, 2015.
30 Lewis JT, Ketterling RP, Halling KC, Reynolds C, Jenkins RB and Visscher DW: Analysis of intratumoral heterogeneity and amplification status in breast carcinomas with equivocal $(2+)$ HER2 immunostaining. Am J Clin Pathol 124(2): 273-281, 2005.

31 Hoang MP, Sahin AA, Ordonez NG and Sneige N: Her-2/neu gene amplification compared with HER2/neu protein overexpression and interobserver reproducibility in invasive breast carcinoma. Am J Clin Pathol 113(6): 852-859, 2000.

32 Bethune GC, Mullen JB and Chang MC: HER2 testing of multifocal invasive breast carcinoma: How many blocks are enough? Am J Clin Pathol 140(4): 588-592, 2013.

33 Buggi F, Folli S, Curcio A, Casadei-Giunchi D, Rocca A, Pietri E, Medri L and Serra L: Multicentric/multifocal breast cancer with a single histotype: Is the biological characterization of all individual foci justified? Ann Oncol 23(8): 2042-2046, 2012.

34 Choi Y, Kim EJ, Seol H, Lee HE, Jang MJ, Kim SM, Kim JH, Kim SW, Choe G and Park SY: The hormone receptor, human epidermal growth factor receptor 2, and molecular subtype status of individual tumor foci in multifocal/multicentric invasive ductal carcinoma of breast. Hum Pathol 43(1): 48-55, 2012.

35 Apple SK, Lowe AC, Rao PN, Shintaku IP and Moatamed NA: Comparison of fluorescent in situ hybridization HER2/neu results on core needle biopsy and excisional biopsy in primary breast cancer. Mod Pathol 22(9): 1151-1159, 2009.

36 Jourdan F, Sebbagh N, Comperat E, Mourra N, Flahault A, Olschwang S, Duval A, Hamelin R and Flejou JF: Tissue microarray technology: Validation in colorectal carcinoma and analysis of p53, hMLH1, and hMSH2 immunohistochemical expression. Virchows Arch 443(2): 115-121, 2003.

37 Khouja MH, Baekelandt M, Sarab A, Nesland JM and Holm R: Limitations of tissue microarrays compared with whole-tissue sections in survival analysis. Oncol Lett 1(5): 827-831, 2010.

38 Conway C, Dobson L, O'Grady A, Kay E, Costello S and O'Shea D: Virtual microscopy as an enabler of automated/ quantitative assessment of protein expression in TMAs. Histochem Cell Biol 130(3): 447-463, 2008.

39 Gancberg D, Di Leo A, Rouas G, Jarvinen T, Verhest A, Isola J, Piccart MJ and Larsimont D: Reliability of the tissue microarray based FISH for evaluation of the HER 2 oncogene in breast carcinoma. J Clin Pathol 55(4): 315-317, 2002.

Received March 31, 2017

Revised April 16, 2017

Accepted April 19, 2017 